NASA Technical Memorandum 106614

ICOMP-94-09; CMOTT-94-03

AIAA-94-3356

\title{
Comparison of PDF and Moment Closure Methods in the Modeling of Turbulent Reacting Flows
}

Andrew T. Norris

Institute for Computational Mechanics in Propulsion

and Center for Modeling of Turbulence and Transition

National Aeronautics and Space Administration

Lewis Research Center

Cleveland, Ohio

and

Andrew T. Hsu

Center for Modeling of Turbulence and Transition

NYMA, Inc.

Engineering Services Division

Brook Park, Ohio

Prepared for the

30th Joint Propulsion Conference cosponsored by AIAA, ASME, SAE, and ASEE Indianapolis, Indiana, June 27-29, 1994

National Aeronautics and

Space Administration

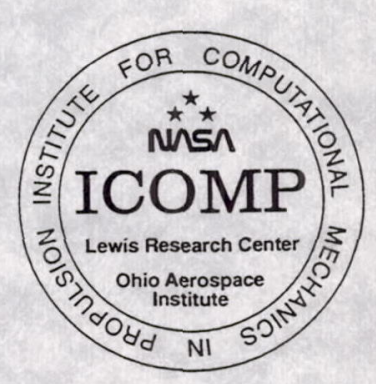




\title{
COMPARISON OF PDF AND MOMENT CLOSURE METHODS IN THE MODELING OF TURBULENT REACTING FLOWS
}

\author{
Andrew T. Norris \\ Institute for Computational Mechanics in Propulsion \\ and Center for the Modeling of Turbulence and Transition \\ National Aeronautics and Space Administration \\ Lewis Research Center \\ Cleveland, Ohio 44135 \\ Andrew T. Hsu \\ Center for Modeling of Turbulence and Transition \\ and NYMA, Inc. \\ Engineering Services Division \\ Brook Park, Ohio 44142
}

\begin{abstract}
In modeling turbulent reactive flows, PDF (Probability Density Function) methods have an advantage over the more traditional moment closure schemes in that the PDF formulation treats the chemical reaction source terms exactly, while moment closure methods are required to model the mean reaction rate. The common model used is the laminar chemistry approximation, where the effects of turbulence on the reaction are assumed negligible. For flows with low turbulence levels and fast chemistry, the difference between the two methods can be expected to be small. However for flows with finite rate chemistry and high turbulence levels, significant errors can be expected in the moment closure method.

In this paper, the ability of the PDF method and the moment closure scheme to accurately model a turbulent reacting flow is tested. To accomplish this, both schemes were used to model a $\mathrm{CO} / \mathrm{H}_{2} / \mathrm{N}_{2^{-}}$ air piloted diffusion flame near extinction. Identical thermochemistry, turbulence models, initial conditions and boundary conditions are employed to ensure a consistent comparison can be made. The results of the two methods are compared to experimental data as well as to each other. The comparison reveals that the PDF method provides good agreement with the experimental data, while the moment closure scheme incorrectly shows a broad, laminarlike flame structure.
\end{abstract}

\section{Introduction}

Chemically reacting flows are described by the continuity, momentum, energy, and species transport equations:

$$
\begin{aligned}
\frac{D \rho}{D t} & =-\rho \frac{\partial U_{i}}{\partial x_{i}} \\
\rho \frac{D U_{i}}{D t} & =-\frac{\partial p}{\partial x_{i}}+\frac{\partial \tau_{i j}}{\partial x_{j}}+\rho g_{i} \\
\rho \frac{D h}{D t} & =-\frac{\partial q_{j}}{\partial x_{j}}+\frac{D p}{D t}+Q+\Phi \\
\rho \frac{D \phi_{\alpha}}{D t} & =-\frac{\partial J_{i}^{\alpha}}{\partial x_{i}}+\rho S_{\alpha}
\end{aligned}
$$

where the material derivative is

$$
\frac{D}{D t}=\frac{\partial}{\partial t}+U_{j} \frac{\partial}{\partial x_{i}},
$$

$\rho$ is the density, $U_{i}$ is the velocity, $p$ is the pressure, $g_{i}$ is the body force and $\tau_{i j}$ is the stress tensor. The specific enthalpy is denoted by $h, q_{i}$ is the diffusive flux of enthalpy, $Q$ is the enthalpy source term and $\Phi$ is the viscous dissipation. In the species transport equation, $\phi_{\alpha}$ is a scalar quantity, (e.g. the mass fraction), $J_{i}^{\alpha}$ is the diffusive flux of $\phi_{\alpha}$ and $S_{\alpha}$ is the scalar source term, also called the reaction rate. An equation of state is also required to close this system of equations. In the above equations it is important to note that the chemical source term, 
$S_{\alpha}$, is uniquely determined by the composition and state variables. Thus the reaction rate can be given by

$$
S_{\alpha}=\hat{S}_{\alpha}(\underline{\phi}, p, h),
$$

where $\phi$ is the vector of $\sigma$ different species variables. For laminar flows, this system of equations can be solved directly. However for all but the simplest turbulent flows, this is not possible, and so a different approach is required.

For the past 20 years, the mean flow or moment closure method has been the principal tool used solve this system of equations for turbulent flows. In this method, the flow variables are decomposed into mean and fluctuating components, and the conservation equations for the mean quantities are obtained. The unknown second order terms that appear in these mean conservation equations are modeled (eg. $k-\epsilon$ model) so as to affect closure. However the modeling of the mean chemical source term, $\left\langle S_{\alpha}\right\rangle$, proves to be extremely difficult. The most common method used is the laminar chemistry approximation, where it is assumed that the mean reaction rate is equal to the reaction rate of the mean composition and state variables:

$$
\left\langle S_{\alpha}\right\rangle \approx \hat{S}_{\alpha}\left(\left\langle\phi_{1}\right\rangle,\left\langle\phi_{2}\right\rangle, \ldots,\left\langle\phi_{\sigma}\right\rangle,\langle p\rangle,\langle h\rangle\right) .
$$

However due to the highly non-linear nature of the reaction rates, this approximation can be in error by several orders of magnitude [1]. The only conditions where this approximation can be considered valid are for low-turbulence, fast-reacting flows.

In the past few years, the development of the PDF (Probability Density Function) method has provided an alternate approach to calculating turbulent reacting flows [2]. The PDF method involves solving the transport equation for the joint $\mathrm{PDF}$ of the flow variables, rather than just their mean value. One of the attractions of using a PDF method to model turbulent combustion is that the reaction term is treated exactly $[3,2,1]$, thus providing a method of modeling reacting flows where strong turbulent/chemical interactions occur.

The purpose of this paper is twofold. First we wish to demonstrate the type of errors that result from the laminar chemistry approximation of the mean reaction rate in a real flow. Secondly we want to show how the PDF method can accurately calculate flows with high turbulence-chemistry interactions, where moment closure methods with the laminar chemistry approximation can be expected to fail. To achieve these goals, both a moment-closure scheme and a PDF method are used to calculate a turbulent reacting flow. The flow chosen is a piloted $\mathrm{CO} / \mathrm{H}_{2} / \mathrm{N}_{2}$-air diffusion flame investigated by Masri et al [4]. The chemical reaction rates are obtained from a threescalar reduced mechanism, created by the ILDM (Intrinsic Low-Dimensional Manifold) method of Maas and Pope $[5,6]$. The PDF method chosen is a compressible hybrid scheme, $[7,8]$ with the velocity field solved by a moment closure method, and the scalar field by a particle-based PDF scheme.

\section{Hybrid PDF Method}

The hybrid PDF scheme is a combination of moment closure and PDF methods. The continuity and momentum equations are solved by a moment closure method while the transport equation for the joint PDF of enthalpy and composition is used to solve the scalar and energy fields. This combination has the advantage in that the PDF part of the code can be added to an existing moment closure method with a minimal amount of coding. Thus existing combustion codes can be extended to include the PDF model of turbulent chemical reactions without the need for extensive rewriting.

Taking the scalar and enthalpy transport equations (Eqn. 4), (but neglecting $Q$ and $\Phi$,) standard methods $[9,2]$ give the evolution equation of the density weighted, joint PDF of composition $\phi$ and specific enthalpy $h, f_{\phi h}$ :

$$
\begin{aligned}
\frac{\partial}{\partial t}\left(\bar{\rho} f_{\phi h}\right) & +\frac{\partial}{\partial x_{i}}\left(\bar{\rho} \bar{U}_{i} f_{\phi h}\right) \\
& +\frac{\partial}{\partial \psi_{\alpha}}\left(\bar{\rho} \hat{S}_{\alpha}(\underline{\psi}, p, \eta) f_{\phi h}\right) \\
& =\frac{\partial}{\partial x_{i}}\left(\left\langle\bar{\rho} \dot{u}_{i} \mid \underline{\psi}, \eta\right\rangle f_{\phi h}\right) \\
& +\frac{\partial}{\partial \psi_{\alpha}}\left(\left\langle\frac{\partial J_{i}^{\alpha}}{\partial x_{i}} \mid \underline{\psi}, \eta\right\rangle f_{\phi h}\right) \\
& +\frac{\partial}{\partial \eta}\left(\left\langle\frac{\partial q_{i}}{\partial x_{j}} \mid \underline{\psi}, \eta\right\rangle f_{\phi h}\right) \\
& +\frac{\partial}{\partial \eta}\left(\left\langle\frac{D p}{D t} \mid \underline{\psi}, \eta\right\rangle f_{\phi h}\right)
\end{aligned}
$$

where $\psi_{\alpha}$ and $\eta$ are the sample space variables corresponding to $\phi_{\alpha}$ and $h$ respectively, and $\langle a \mid b\rangle$ denotes the expectation of $a$ conditional on $b$. Note that in Eqn. 8 all the terms on the left hand side (LHS) are exact, including the important reaction source term. 
The terms on the right-hand side (RHS) of Eqn. 8 however contain unknown conditional expectations, and so need to be modeled.

The first term on the RHS of Eqn. 8 corresponds to the turbulent convection of the PDF. This is modeled by a simple gradient diffusion model. The next two terms correspond to the molecular mixing of the composition and the enthalpy respectively. These two terms are both modeled by a molecular mixing model [10]. The final term to be modeled is the pressure term, which includes the pressure-dilatation term. For details of the modeling of this and the other terms, readers are referred to Hsu et al [7].

\section{Experiment}

The experimental flow chosen for this study is the $\mathrm{CO} / \mathrm{H}_{2} / \mathrm{N}_{2}$-air flame of Masri et al [4] with a bulk fuel velocity of $98.0 \mathrm{~m} / \mathrm{s}$. This flame was chosen as it has mild compressibility effects, finite rate chemistry and high levels of turbulence.

The apparatus consists of a central fuel jet of $7.2 \mathrm{~mm}$ inside diameter, surrounded by an annulus of $18.0 \mathrm{~mm}$ outside diameter, containing the pilot flame. This jet assembly is mounted at the center of the exit plane of a $305.0 \times 305.0 \mathrm{~mm}$ wind tunnel, which produces a uniform, low turbulence intensity flow of air with a velocity of $15.0 \mathrm{~m} / \mathrm{s}$. The fuel composition is $45 \% \mathrm{CO}, 15 \% \mathrm{H}_{2}$ and $40 \% \mathrm{~N}_{2}$ by volume, and the pilot flame consists of a lit, premixed flow of fuel and air in stoichiometric proportions, with an unlit bulk velocity of $1.0 \mathrm{~m} / \mathrm{s}$.

Data from the experiment consists of the joint PDFs of composition taken at a point $72.0 \mathrm{~mm}$ downstream of the jet exit plane, and approximately $6.5 \mathrm{~mm}$ out from the axis. Note that this radial location was chosen to correspond with the peak mean radial temperature of the flame. These PDFs were obtained by the Rayleigh-Raman scattering technique, with 2000 composition samples. Extinction for this flame occurs at a fuel-jet bulk velocity of $167.0 \mathrm{~m} / \mathrm{s}$.

\section{Thermochemistry}

The ILDM (Intrinsic Low-Dimensional Manifold) method of Maas and Pope [5, 6] was used to generate the thermochemical model used in this study. The ILDM method is a general procedure for simplifying chemical kinetics, based on a dynamical systems



Figure 1: Example of tabulated properties. Section taken at $\xi=0.37$, shows reaction rate of $\mathrm{CO}_{2}$ as a function of of $\mathrm{CO}_{2}$ and $\mathrm{H}_{2} \mathrm{O}$. Units are mole $/ \mathrm{kg}$.

approach, requiring only the full chemical mechanism and the dimension, $n_{r}$ of the resulting reduced mechanism as input. Given the inputs, the ILDM scheme identifies the time scale of each reaction for all allowed compositions. By freezing the $n_{r}$ slowest reactions and letting the rest relax to equilibrium, a low-dimensional attracting manifold is obtained. This manifold is then projected onto a set of $n_{r}$ species, referred to as the controlling variables. The rate of change of these controlling variables is known at all points on the manifold, completing the creation of the reduced mechanism.

For the $\mathrm{CO} / \mathrm{H}_{2} / \mathrm{N}_{2}$-air system used in this study, the full mechanism and details of the ILDM method are described by Maas and Pope [5]. A three-scalar mechanism was obtained from the full system, with the three representative scalars being the mixture fraction $\xi$, and the mass fractions of $\mathrm{CO}_{2}$ and $\mathrm{H}_{2} \mathrm{O}$, denoted by $\mathrm{YCO}_{2}$ and $Y_{\mathrm{H}_{2} \mathrm{O}}$ respectively. The mixture fraction is a conserved scalar based on the mass fraction of $\mathrm{N}_{2}$, with a value of zero for pure air and unity for pure fuel. The reduced mechanism is stored in the form of composition increments due to reaction for given time intervals and specified initial composition. An adaptive tabulation method is used to store these increment tables. It should be noted that as $\xi$ is a conserved scalar, only the increments 


\begin{tabular}{|l|c|c|}
\hline & $d Y_{\mathrm{CO}_{2}} / d t$ & $d Y_{\mathrm{H}_{2} \mathrm{O}} / d t$ \\
\hline Approx & 24.1 & -0.62 \\
\hline PDF & 4.77 & 0.77 \\
\hline
\end{tabular}

Table 1: Mean reaction rates for an experimentally measured mixture, obtained by the laminar chemistry approximation and the PDF method. Units are in mass fractions per second.

of $Y_{\mathrm{CO}_{2}}$ and $Y_{\mathrm{H}_{2} \mathrm{O}}$ need be stored. Figure 1 shows a section of the reaction increment table for several different properties.

\section{Reaction Rate Errors}

In this section, the error associated with the approximation of the mean reaction rate (Eqn. 7) is investigated. Taking the experimental data as an initial condition, the mean composition is calculated and the mean reaction rate obtained. This rate corresponds to the laminar chemistry approximation of the mean reaction rate employed in moment closure schemes. For the exact mean reaction rate, the reaction rate for each of the 2000 sample compositions is obtained, and then the mean is calculated. The results are shown in Tab. 1 . The results show a considerable difference between the reaction rates, with the $Y_{\mathrm{CO}_{2}}$ rates differing by an order of magnitude, while the $Y_{\mathrm{H}_{2} \mathrm{O}}$ rates differ in sign. These results show that the approximation of Eqn. 7 can be wrong in not only magnitude but in sign as well.

The effect of evolving the reaction rates with time is also investigated. From the same initial conditions as above, the mean composition is reacted at the mean reaction rate, while each individual sample is reacted by its own reaction rate, and then the new mean composition is calculated. The evolution of $Y_{\mathrm{CO}_{2}}$ and $Y_{\mathrm{H}_{2} \mathrm{O}}$ with time for both reaction methods are shown in Fig. 2.

It can be seen that at first the reaction paths differ considerably, and while the $Y_{\mathrm{H}_{2} \mathrm{O}}$ paths asymptote to approximately the same values, the long-time $\mathrm{Y}_{\mathrm{CO}_{2}}$ values differ by about $20 \%$. This difference is caused by the non-linear nature of the reaction equations combined with the large variation in the initial sample composition, caused by the high level of turbulent mixing. The implication of these results is that
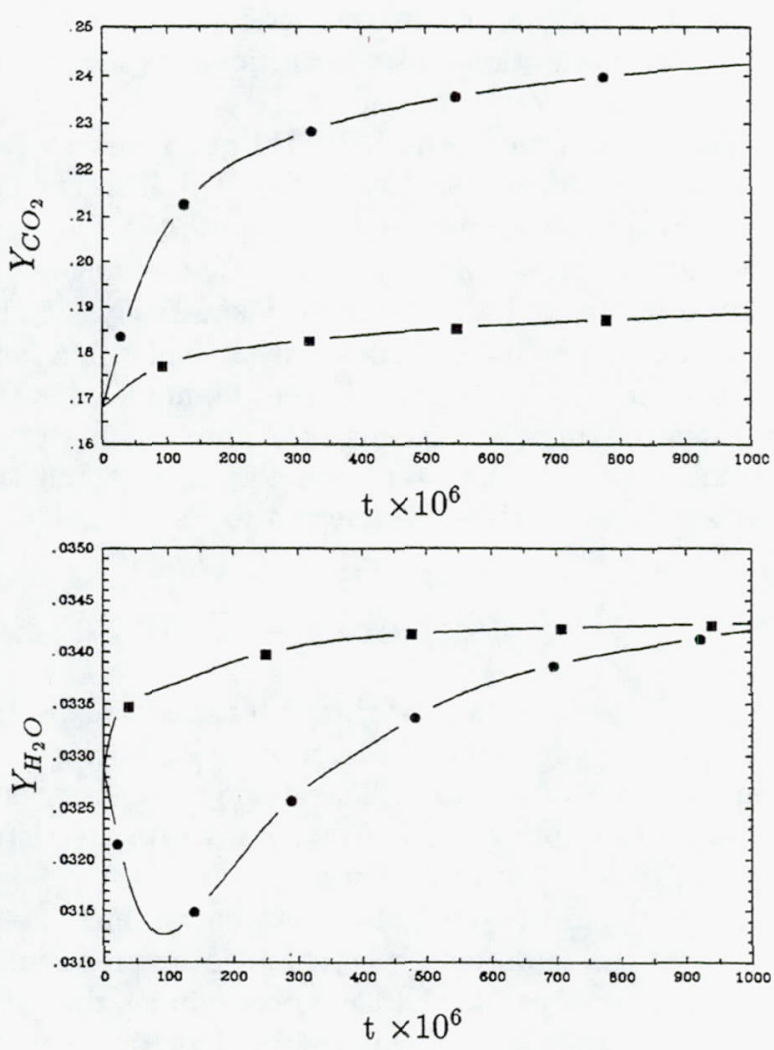

Figure 2: Reaction trajectories for $Y_{\mathrm{CO}_{2}}$ and $Y_{\mathrm{H}_{2} \mathrm{O}}$ obtained by the laminar chemistry approximation and the PDF method. $O$ : Laminar approximation; $\square$ : PDF result. Units are mass fractions and seconds.

in relatively high turbulence level flows, even very fast, equilibrium-type reactions can be poorly represented by the laminar chemistry approximation.

\section{Flame Calculation}

In this section, the relative abilities of a moment closure scheme and a PDF method are compared when applied to a turbulent reactive flow with finite rate chemistry. The moment closure scheme is the RPLUS code [11], which was developed specifically for high-speed reacting flows. The turbulence model used is the standard $k-\epsilon$ model for high Reynolds number flows, and the reaction source term is modeled by the laminar chemistry approximation. The PDF method is the hybrid PDF scheme of Hsu et al $[7,8]$, which uses the RPLUS code to provide the mean velocity and density field, while the trans- 
port equation for the joint PDF of composition and enthalpy is solved by a particle-based Monte Carlo scheme.

\section{Solution Procedure}

The solution procedure for moment closure and PDF scheme have been documented previously [11, $12,7]$, and so only a brief description of the more important features of the codes are given.

The RPLUS code is a $2 \mathrm{D}$, axisymmetric version that uses a LU-SSOR upwind scheme, with a standard high Reynolds number $k-\epsilon$ model.

The PDF code is the same as that developed by Hsu et al [7], but with a few changes. These changes primarily involve the treatment of the convection, molecular mixing and the averaging process. The convection of scalar particles has been altered to account for the convection of fractions of a particle. At each step, a whole number of particles are convected, and any remainder is carried on to the next step. Molecular mixing is carried out by the Relax-toMean model of Dopazo [10], rather than the model by Hsu and Chen [13]. This change was made to provide a faster performance, and allow a larger timestep to be used in the calculations. The averaging process has been changed to provide a more efficient use of computer memory. The method employed is the weighted average scheme [8]. In this method, the weighted time-average of some mean quantity $\langle\phi\rangle$ at the $n$th time step is given by:

$$
\langle\bar{\phi}\rangle_{n}=\frac{1}{w_{n}+1}\left(\langle\phi\rangle_{n}+w_{n}\langle\bar{\phi}\rangle_{n-1}\right),
$$

where the over-bar indicates a time averaged quantity and $w_{n}$ is a weighting function,

$$
w_{n}=c_{n}\left(w_{n-1}+1\right) \text {. }
$$

The variable $c_{n}$ is chosen to have a value of 0.9 for $n \leq 2000,0.999$ for $n \geq 5000$ and a linear variation between these two values. These values were chosen to provide a quick response time for the average values while the flow computation approaches a steady-state solution, and then changing to minimize the statistical fluctuation of the average, allowing the solution to converge further.

\section{Initial Conditions}

The quantities solved for by both solution schemes are the density, mean axial velocity, mean radial ve- locity, turbulent kinetic energy, dissipation rate and internal energy. The composition variables are the mass fractions of $\mathrm{H}_{2}, \mathrm{O}_{2}, \mathrm{H}_{2} \mathrm{O}, \mathrm{CO}, \mathrm{CO}_{2}$ and $\mathrm{N}_{2}$. In addition the PDF code solves for the specific enthalpy of the flow.

The initial conditions at the jet exit plane are the combination of three different flows, the jet, the pilot and the co-flow.

For the jet, the mass flow rate and the composition are known from the experiment. The initial velocity distribution is obtained by assuming fully developed pipe flow, with the mean velocity, kinetic energy and dissipation profiles taken from Hinze [14].

The mass flow rate and the pre-burned composition of the pilot are known from the experiment. By assuming the pilot to be fully burned at the jet exit, the mean composition, temperature and density are known. The low axial velocity of the pilot and the high temperature result in a Reynolds number of order 100, thus a uniform velocity is assumed. The kinetic energy and dissipation are set close to zero.

For the co-flow, the mean and fluctuating values of the axial velocity are known and correspond to a turbulent boundary layer on the outside wall of the pilot jet. So as in the jet, the mean velocity distribution, kinetic energy and dissipation are taken from Hinze [14].

The computational domain is rectangular, extending 20 jet diameters downstream and 7.5 jet diameters out from the axis. The solution grid uses 50 cells in the axial and radial direction, with a higher density of cells in regions around the jet exit. Boundary conditions are an axis of symmetry for the center of the jet, and free stream conditions at the outer edge of the flow, corresponding to the known co-flow properties.

\section{Results}

In this section the results of the two schemes are compared to experimental data and each other. Data includes convergence histories, mean velocity fields, mean scalar fields and scatter-plots for the PDF results. The differences between the two computational schemes, and the experimental data are then discussed.

\section{Errors and Convergence}

The statistical error in evaluating the mean of a scalar is estimated by taking the average scalar value 


\begin{tabular}{|c|c|}
\hline Quantity & Error \\
\hline$\left\langle Y_{\mathrm{CO}_{2}}\right\rangle$ & $\pm 1.14 \%$ \\
\hline$\left\langle Y_{\mathrm{H}_{2} \mathrm{O}}\right\rangle$ & $\pm 1.34 \%$ \\
\hline$\langle T\rangle$ & $\pm 0.83 \%$ \\
\hline
\end{tabular}

Table 2: $95 \%$ confidence limits for the fluctuation of time-averaged scalar quantities used in the PDF method.

at a point every 100 time-steps, and obtaining the fluctuation of this quantity. The point chosen for this test corresponds to the point where the experimental data was obtained, and 30 samples were taken. The $95 \%$ confidence limits in percent of the mean scalar value are shown in Tab. 2.

Due to the statistical fluctuation associated with the mean scalar value in PDF methods, the concept of convergence is different to that of moment closure convergence. The residual error at time-step $n, \varepsilon_{n}(\langle\bar{\phi}\rangle)$, of some averaged quantity, $\langle\bar{\phi}\rangle_{n}$, can be defined as;

$$
\varepsilon_{n}(\langle\bar{\phi}\rangle) \equiv \frac{1}{N x} \frac{1}{N r} \sum_{i=1}^{N x} \sum_{j=1}^{N r} \frac{\left(\left\langle\overline{\phi_{i j}}\right\rangle_{n}-\left\langle\overline{\phi_{i j}}\right\rangle_{n-1}\right)^{2}}{\left\langle\bar{\phi}_{i j}\right\rangle_{n}^{2}}
$$

where $N x$ and $N r$ are the number of cells in the axial $(x)$ and radial $(r)$ directions respectively. For moment closure methods, convergence is achieved when $\varepsilon_{n}(\langle\bar{\phi}\rangle)$ is reduced to the level of machine, or roundoff error. For PDF methods, convergence is achieved when $\varepsilon_{n}(\langle\bar{\phi}\rangle)$ is reduced to an acceptable level of statistical fluctuation of the solution. This level is determined by the number of particles in each cell and the amount of time-averaging made to the solution. Figure 3 shows the error history for the mass fractions of $\mathrm{CO}_{2}$ and $\mathrm{H}_{2} \mathrm{O}$. The shape of the error curves are similar and demonstrate the imprecise nature of PDF convergence. After approximately 1000 steps, the error stops decreasing, corresponding to the steady state for the small amount of time averaging with $c_{n}=0.9$ in Eqn. 10. Between 2000 and 5000 steps, $c_{n}$ is increased to a value of 0.999 and the errors show a steep decent, reflecting the lowering of the statistical uncertainty in the mean values. Between 5000 and 10000 steps, the errors reach a new steady state, corresponding to a converged solution for $c_{n}=0.999$.

The overall rate of convergence for this problem is rather slow due to the RPLUS code being used to
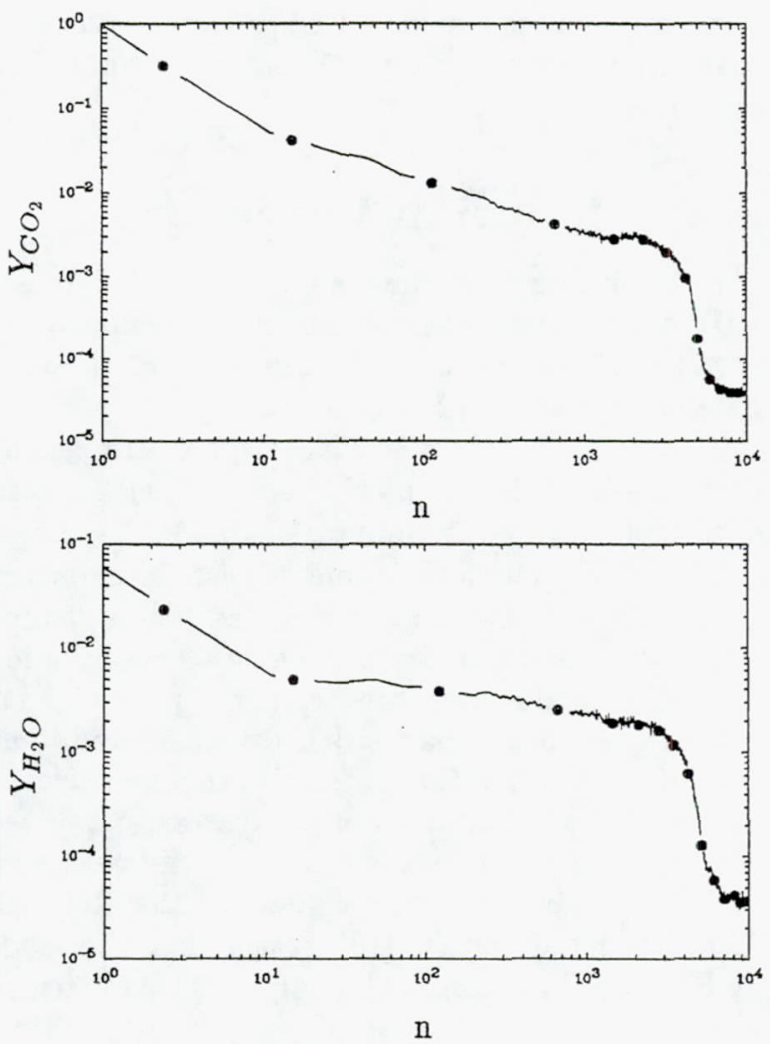

Figure 3: Convergence histories for the residual error of $Y_{\mathrm{CO}_{2}}$ and $Y_{\mathrm{H}_{2} \mathrm{O}}$ produced by the PDF method.

model a low-speed flow. Much faster convergence is obtainable with this code for supersonic flows [7].

\section{Mean Flow Fields}

In Tab. 3 the mean mass fractions of $\mathrm{CO}_{2}$ and $\mathrm{H}_{2} \mathrm{O}$ are shown, as well as the mixture fraction and temperature. These values are obtained from the moment closure, PDF and experiment, at a location $72.0 \mathrm{~mm}$ downstream of the jet exit plane and 6.5 $\mathrm{mm}$ out from the axis. The comparison between the experimental data and the PDF results are generally in good agreement. The underprediction of $\left\langle Y_{\mathrm{H}_{2} \mathrm{O}}\right\rangle$ is thought to be caused by the neglection of the water vapor content of the air in the calculations and the assumed equal diffusivities of species in the PDF method. The moment closure results at this location also agree reasonably well with the experimental data, except for the value of $Y_{\mathrm{CO}_{2}}$ which is underpredicted. The value of $Y_{\mathrm{H}_{2} \mathrm{O}}$ shows a similar level of underprediction as the PDF result, and the 


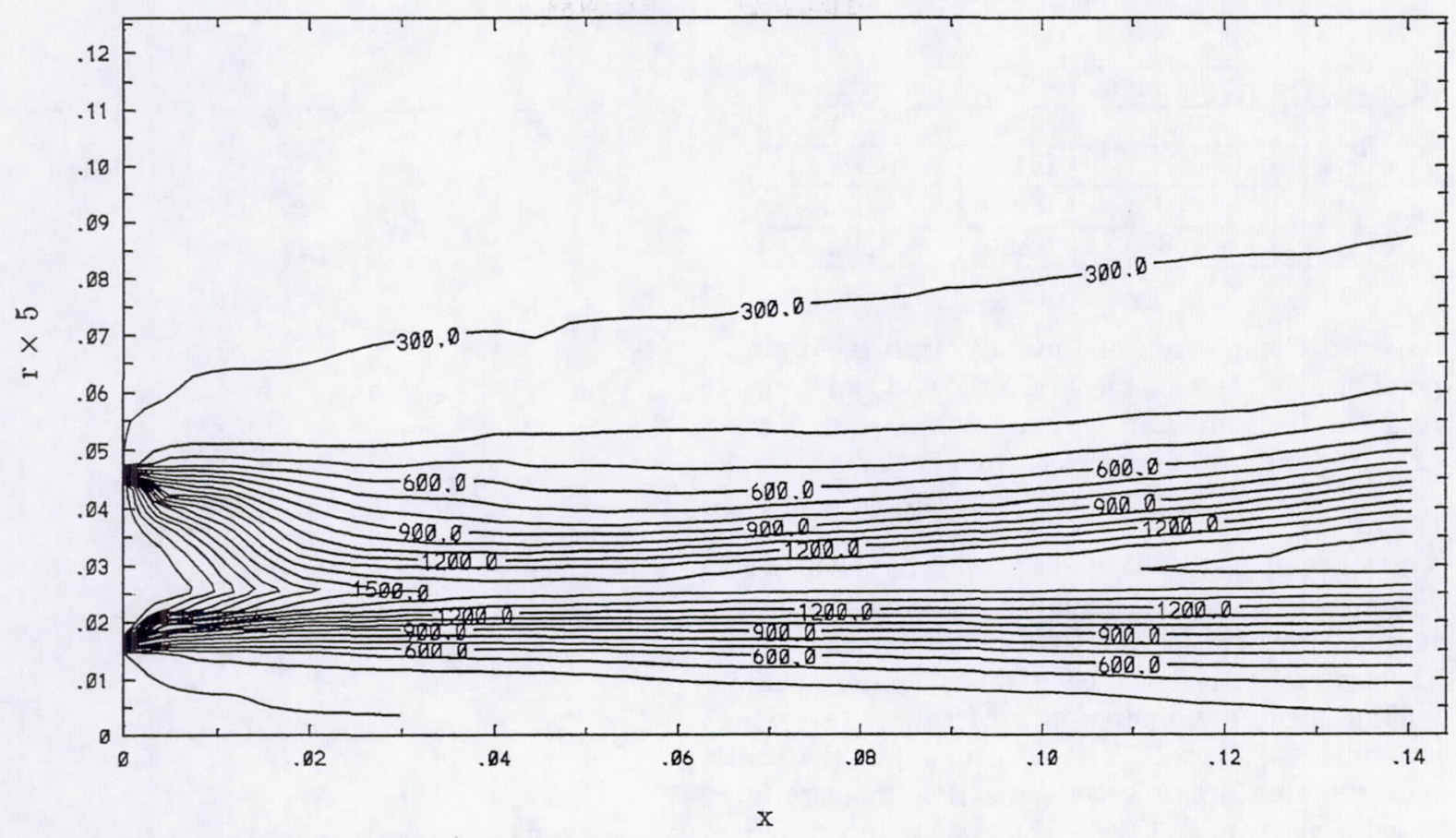

Figure 4: Contour plots of mean flame temperature produced by the PDF method. Note that radial coordinate is stretched by factor of five. Contour levels are in degrees Kelvin.

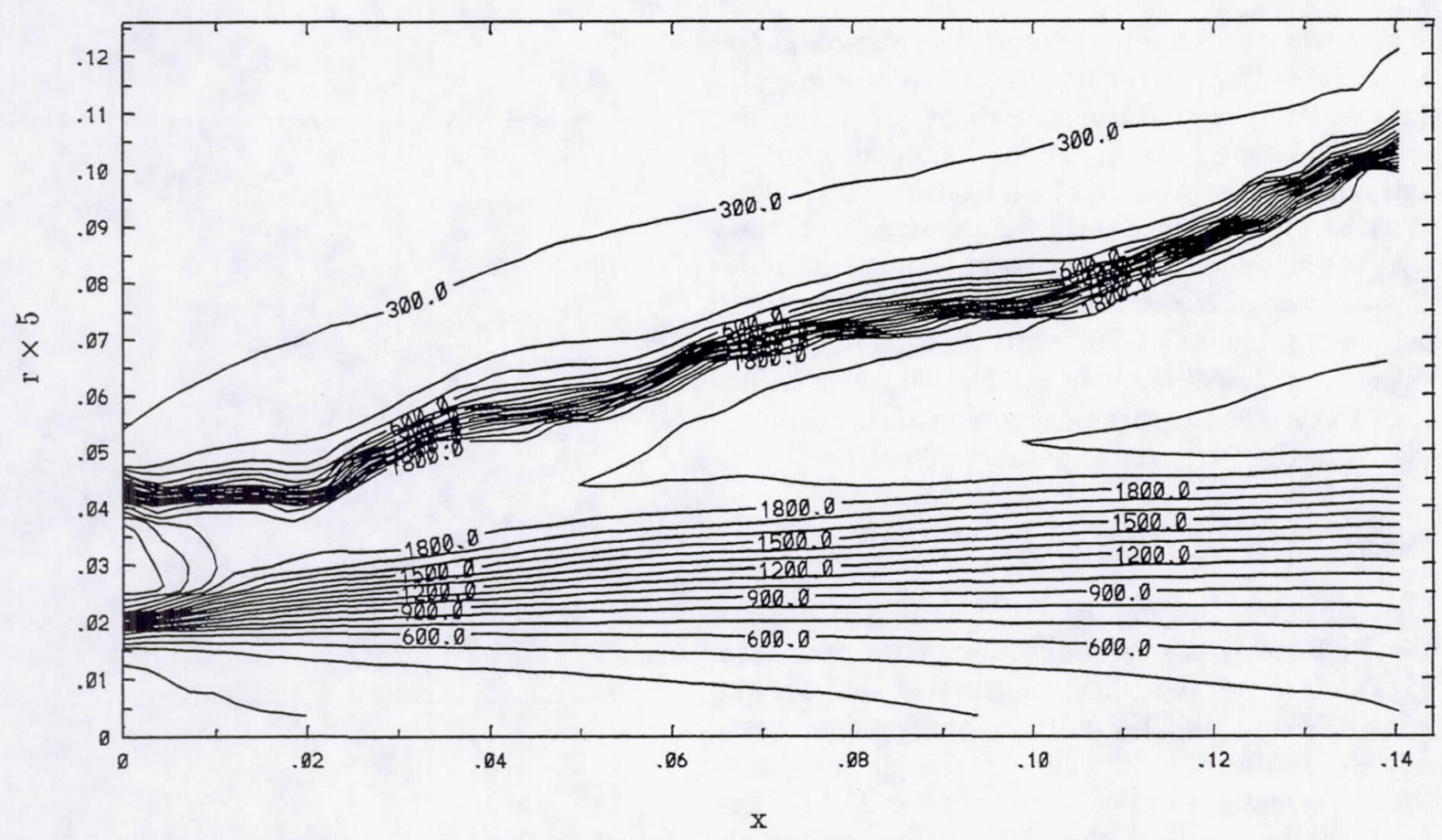

Figure 5: Contour plots of mean flame temperature produced by the moment closure method. Note that radial coordinate is stretched by factor of five. Contour levels are in degrees Kelvin. 


\begin{tabular}{|l|c|c|c|}
\hline & Experiment & PDF & Experiment \\
\hline$\langle\xi\rangle$ & 0.32 & 0.31 & 0.33 \\
\hline$\left\langle Y_{\mathrm{CO}_{2}}\right\rangle$ & 0.17 & 0.158 & 0.121 \\
\hline$\left\langle Y_{\mathrm{H}_{2} \mathrm{O}}\right\rangle$ & 0.033 & 0.024 & 0.022 \\
\hline$\langle T\rangle$ & 1400 & 1380 & 1500 \\
\hline
\end{tabular}

Table 3: Comparison of time averaged mean flow quantities for moment closure, PDF and experimental data. Data location is $72 \mathrm{~mm}$ downstream of the jet exit plane, and $6.5 \mathrm{~mm}$ out from the axis.

mean temperature is slightly overpredicted. Figures 4 and 5 show the contour plots of the mean temperature field for the PDF and moment closure schemes respectively. The PDF calculation shows a flame structure similar in structure to previous experiments [15], with a rapid drop-off in the peak axial temperature, followed by a slow increase further downstream and the slight "waist" in the flame width. At the experimental data location, the peak radial temperature can also be seen to occur approximately at the experimentally determined radius of $6.5 \mathrm{~mm}$.

However the moment closure calculation shows a significantly different form. At the experimental data location, the peak mean radial temperature lies in a broad band between 9 and $12 \mathrm{~mm}$ out from the axis, and be overpredicted by approximately $500 \mathrm{de}$ grees $\mathrm{K}$. The structure of the temperature field shows that the moment closure results predict a much more rapid spread of the reacting fluid, and the steep temperature gradients at the interface with the co-flow suggest an almost laminar flame sheet. This is contrast to the diffuse, turbulent combustion regime observed in the PDF and experimental results.

\section{Scalar Joint PDFs}

Figure 6 shows the joint PDF of $\xi$ and $Y_{\mathrm{CO}_{2}}$ for both the PDF calculations and the experimental data. Figure 7 shows the same information, but for the joint PDF of $\xi$ and $Y_{\mathrm{H}_{2} \mathrm{O}}$. It is apparent from these figures, despite the difference in sample sizes, that the PDF method has captured the form of the experimental data. Both the PDF and the experimental results lie near the equilibrium curve, and show no evidence of extinction. The high level of scatter in the experimental results can be attributed in part to differential diffusion, a process neglected by the

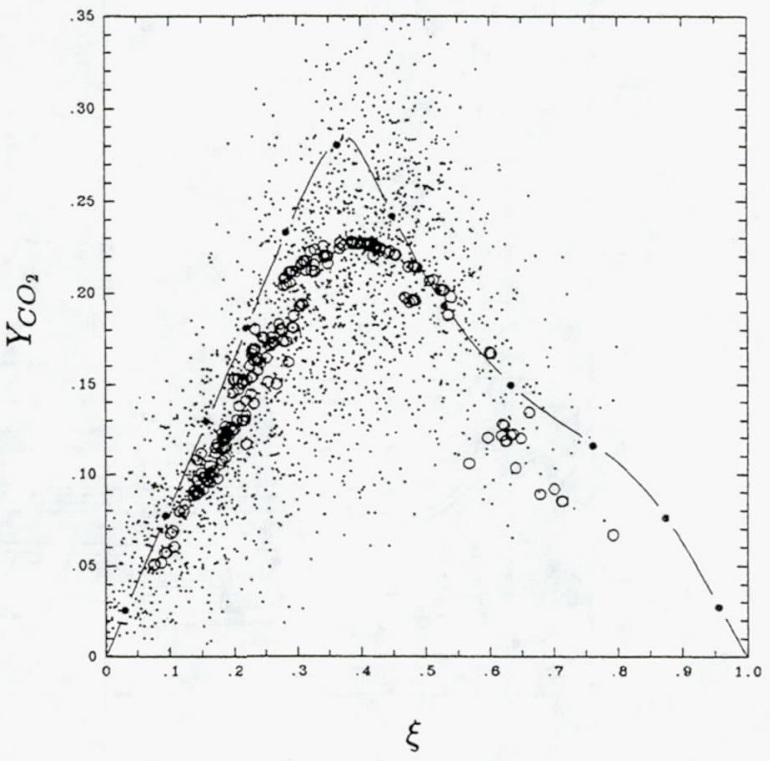

Figure 6: Scatter plot showing the joint PDF of $\xi$ and $\mathrm{Y}_{\mathrm{CO}_{2}}$. Open symbols are PDF results, points are experimental data and the solid line is the equilibrium curve.

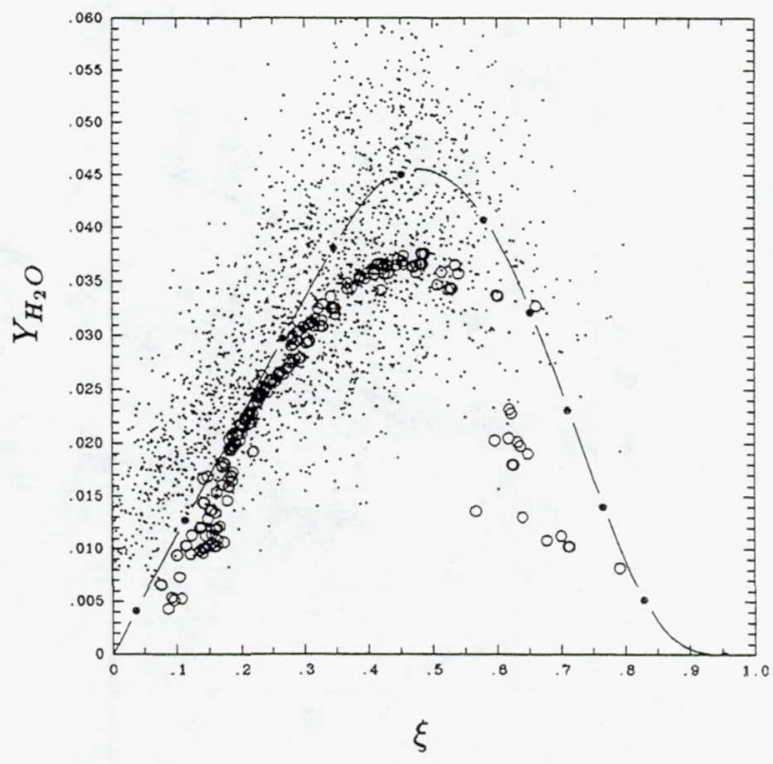

Figure 7: Scatter plot showing the joint PDF of $\xi$ and $Y_{\mathrm{H}_{2} \mathrm{O}}$. Open symbols are PDF results, points are experimental data and the solid line is the equilibrium curve. 
PDF calculation, and experimental error.

The magnitude of the error due to experimental inaccuracies and differential diffusion of species can be illustrated by considering the joint PDF of mixture fraction and $\mathrm{N}_{2}$ shown in Fig. 8. For the case of equal species diffusivities, and the absence of experimental error, all the data points should lie on the straight line. However it is apparent that this is not the case, and that there is a somewhat large degree of uncertainty to the experimental error. It should be noted though, that the expected value of $Y_{N_{2}}$ does lie close to the equal diffusivity line.

The effect of neglecting the water vapor in the coflow is apparent on the lean side of the stoichiometric mixture fraction of Fig. 7, where the experimental points show a higher value than the PDF results and the equilibrium line.

A similar comparison can not be made between the moment closure results, and the experimental data as only the mean scalar values are available, and so would be represented by a single point in Figs. 6 and 7 .

Similar joint PDF's to those in Fig. 6 and 7 for the same flame configuration have been obtained by Norris and Pope [16, 17] using a parabolic Monte Carlo scheme to solve the transport equation for the joint PDF of velocity, dissipation and composition. In their study, the fuel jet velocity was increased until the PDF model predicted that the flame had been extinguished. Excellent agreement was found between the experimental and PDF results, with extinction being predicted within $5 \%$ of the experimental value. Similar tests have not yet been performed with the hybrid PDF model.

\section{Conclusion}

The relative ability of a moment closure scheme and a hybrid PDF method to model a $\mathrm{CO} / \mathrm{H}_{2} / \mathrm{N}_{2}$ air piloted turbulent diffusion flame near extinction has been evaluated.

First the magnitude of the error associated with the laminar chemistry approximation of the mean reaction source term was investigated. It was shown that the approximation of the mean reaction rate can be in error by an order of magnitude, and that the sign of the term can also be wrong. The mean reaction trajectories in scalar-space were also obtained and showed that while the reaction rate approximation also leads to errors in the equilibrium compo-

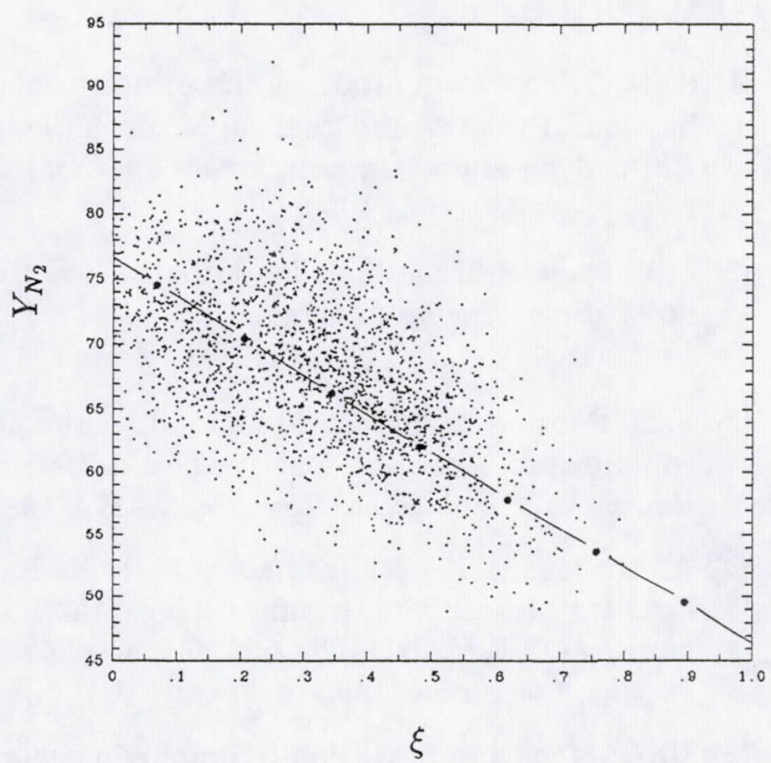

Figure 8: Scatter plot showing the joint PDF of $\xi$ and $Y_{N_{2}}$. Straight line is the result for equal species diffusivities and no experimental error. Points are experimental data.

sitions, these errors are an order of magnitude less than the reaction rate errors.

The performance comparison of the moment closure and PDF method showed the superior ability of the PDF method to accurately simulate a real combustion problem. While the PDF method predicted a turbulent flame structure, with peak mean radial temperature agreeing with experimental value both in location and magnitude, the moment closure method showed a laminar-type flame structure, with the peak mean radial temperature in the wrong location, and overpredicted by 500 degrees $\mathrm{K}$.

\section{Acknowledgements}

The authors would like to thank A. Masri for the use of his experimental data, U. Maas for the ILDM reduced mechanism used in the PDF calculations and B. Duncan for assistance with the RPLUS code. 


\section{References}

[1] S. B. Pope. Computations of turbulent combustion: Progress and challenges. In TwentyThird Symposium (International) on Combustion, pages 591-611, 1990.

[2] S. B. Pope. Pdf methods for turbulent reactive flows. Prog. Energy Combust. Sci., 11:119-192, 1985.

[3] S. B. Pope. A Monte Carlo method for the pdf equations of turbulent reactive flow. Combustion Science and Technology, 25:159-174, 1981.

[4] A. R. Masri, R. W. Dibble, and R. S. Barlow. The structure of turbulent, pilot-Stabilised flames of $\mathrm{CH}_{4} / \mathrm{CO} / \mathrm{H}_{2} / \mathrm{N}_{2}$ fuel mixtures. Combustion and Flame, 1993. in press.

[5] U. A. Maas and S. B. Pope. Simplifying chemical kinetics: Intrinsic low-dimensional manifolds in composition space. Combustion and Flame, 88(3/4):239-264, 1992.

[6] U. A. Maas and S. B. Pope. Implimentation of simplified chemical kinetics based on intrinsic low-dimensional manifolds. In TwentyFourth Symposium (International) on Combustion, pages 103-112, 1992.

[7] A. T. Hsu, Y. L. P. Tsai, and M. S. Raju. A pdf approach for compressible turbulent reacting flows. In AIAA 31st Aerospace Sciences Meeting and Exhibit, Reno, Nevada, pages AIAA93-0087, 1993.

[8] A. T. Hsu, M. S. Raju, and A. T. Norris. Application of a PDF method to compressible turbulent reacting flows. In AIAA 32nd Aerospace Sciences Meeting and Exhibit, Reno, Nevada, pages AIAA-94-0781, 1994.

[9] S. B. Pope. Transport equation for the joint probability density function of velocity and scalars in turbulent flow. Phys. Fluids, 24(4):588-596, 1981.

[10] C. Dopazo. Pdf approach for a turbulent axisymmetric heated jet: Centerline evolution. Phys. Fluids., 18(4):397-404, 1975.

[11] S. Shuen and S. Yoon. Numerical study of chemically reacting flows using a lower-upper symmetric successive overrelaxation scheme. AIAA, 27 (12):1752-1760, 1989.
[12] A. T. Hsu. A study of hydrogen diffusion flames using PDF turbulence model. In AIAA 22nd Fluid Dynamics, Plasma Dynamics and Lasers Conference, Honolulu, Hawaii, pages AIAA91-1780, 1991.

[13] A. T. Hsu and J. Y. Chen. A continuous mixing model for PDF simulations and its applications to combusting shear flows. In Eighth International Symposium on Turbulent Shear Flows, Munich, Germany, pages 22-4-1 22-4-5, 1991.

[14] J. O. Hinze. Turbulence. McGraw Hill, second edition, 1975.

[15] S. H. Stårner and R. W. Bilger. Characteristics of a piloted diffusion flame designed for study of combustion turbulence interactions. Combustion and Flame, 61:29-38, 1985.

[16] A. T. Norris. The Application of PDF Methods to Piloted Diffusion Flames. PhD thesis, Cornell University, 1993.

[17] A. T. Norris and S. B. Pope. Modeling of extinction in turbulent diffusion flames by the velocity-dissipation-composition PDF method. In Twenty-Fifth Symposium (International) on Combustion, page In Press, 1994. 
Public reporting burden for this collection of information is estimated to average 1 hour per response, including the time for reviewing instructions, searching existing data sources, gathering and maintaining the data needed, and completing and reviewing the collection of information. Send comments regarding this burden estimate or any other aspect of this Davis Highway, Suite 1204, Arlington, VA 22202-4302, and to the Office of Management and Budget, Paperwork Reduction Project (0704-0188), Washington, DC 20503.
1. AGENCY USE ONLY (Leave blank)
2. REPORT DATE
May 1994
3. REPORT TYPE AND DATES COVERED
Technical Memorandum

\title{
4. TITLE AND SUBTITLE \\ Comparison of PDF and Moment Closure Methods in the Modeling \\ of Turbulent Reacting Flows
}

Andrew T. Norris and Andrew T. Hsu

WU-505-90-5K

7. PERFORMING ORGANIZATION NAME(S) AND ADDRESS(ES)

National Aeronautics and Space Administration

Lewis Research Center

Cleveland, Ohio 44135-3191

5. FUNDING NUMBERS

9. SPONSORING/MONITORING AGENCY NAME(S) AND ADDRESS(ES)

10. SPONSORING/MONITORING AGENCY REPORT NUMBER

National Aeronautics and Space Administration

Washington, D.C. 20546-0001

\author{
NASA TM-106614 \\ ICOMP-94-9; CMOTT-94-3 \\ AIAA-94-3356
}

\section{SUPPLEMENTARY NOTES}

Prepared for the 30th Joint Propulsion Conference cosponsored by AIAA, ASME, SAE, and ASEE, Indianapolis, Indiana, June 27-29, 1994. Andrew T. Norris, Institute for Computational Mechanics in Propulsion and Center for Modeling of Turbulence and Transition, NASA Lewis Research Center (work funded by NASA Cooperative Agreement NCC3-233), and Andrew T. Hsu, Center for Modeling of Turbulence and Transition (work funded by NASA Cooperative Agreement NCC3-233), and NYMA, Inc., Engineering Services Division, 2001 Aerospace Parkway, Brook Park, Ohio 44142 (work funded by NASA Contract NAS3-27186). ICOMP Program Director, Louis A. Povinelli, organization code 2600, (216) 433-5818.

12a. DISTRIBUTION/AVAILABILITY STATEMENT 12b. DISTRIBUTION CODE

Unclassified - Unlimited

Subject Category 34

\section{ABSTRACT (Maximum 200 words)}

In modeling turbulent reactive flows, PDF (Probability Density Function) methods have an advantage over the more traditional moment closure schemes in that the PDF formulation treats the chemical reaction source terms exactly, while moment closure methods are required to model the mean reaction rate. The common model used is the laminar chemistry approximation, where the effects of turbulence on the reaction are assumed negligible. For flows with low turbulence levels and fast chemistry, the difference between the two methods can be expected to be small. However for flows with finite rate chemistry and high turbulence levels, significant errors can be expected in the moment closure method. In this paper, the ability of the PDF method and the moment closure scheme to accurately model a turbulent reacting flow is tested. To accomplish this, both schemes were used to model a $\mathrm{CO} / \mathrm{H} 2 / \mathrm{N} 2$ - air piloted diffusion flame near extinction. Identical thermochemistry, turbulence models, initial conditions and boundary conditions are employed to ensure a consistent comparison can be made. The results of the two methods are compared to experimental data as well as to each other. The comparison reveals that the PDF method provides good agreement with the experimental data, while the moment closure scheme incorrectly shows a broad, laminar-like flame structure.

\section{SUBJECT TERMS}

PDF method; Turbulent combustion; Non-premixed combustion

15. NUMBER OF PAGES

\begin{tabular}{l|c}
\hline 17. SECURITY CLASSIFICATION & $\begin{array}{c}\text { 18. SECURITY CLASSIFICATION } \\
\text { OF REPORT }\end{array}$ \\
OF THIS PAGE \\
Unclassified
\end{tabular}

NSN 7540-01-280-5500

\section{SECURITY CLASSIFICATION OF ABSTRACT Unclassified}

20. LIMITATION OF ABSTRACT 